\title{
Alcohol-paired contextual cues produce an immediate and selective loss of goal-directed action in rats
}

\author{
Sean B. Ostlund ${ }^{1,2 *}$, NigelT. Maidment ${ }^{1,2}$ and Bernard W. Balleine $e^{2,3,4}$ \\ Department of Psychiatry and Biobehavioral Sciences, Semel Institute for Neuroscience and Human Behavior, University of California at Los Angeles, Los Angeles, CA, USA \\ 2 The Brain Research Institute, University of California at Los Angeles, Los Angeles, CA, USA \\ ${ }^{3}$ Department of Psychology, University of California at Los Angeles, Los Angeles, CA, USA \\ ${ }_{4}^{4}$ Brain and Mind Research Institute, University of Sydney, Sydney, NSW, Australia
}

\section{Edited by:}

Rui M. Costa, Instituto Gulbenkian de Ciência, Portugal

\section{Reviewed by:}

Henry H. Yin, Duke University, USA

Simon Killcross, The University of New South Wales, Australia

*Correspondence:

Sean B. Ostlund, Department of Psychiatry and Biobehavioral Sciences, University of California at Los Angeles, Semel Institute for Neuroscience and Human Behavior, Los Angeles, CA 90024, USA.

e-mail: sostlund@ucla.edu
We assessed whether the presence of contextual cues paired with alcohol would disrupt rats' capacity to express appropriate goal-directed action control. Rats were first given differential context conditioning such that one set of contextual cues was paired with the injection of ethanol and a second, distinctive set of cues was paired with the injection of saline. All rats were then trained in a third, neutral context to press one lever for grain pellets and another lever for sucrose pellets. They were then given two extinction tests to evaluate their ability to choose between the two actions in response to the devaluation of one of the two food outcomes with one test conducted in the alcohol-paired context and the other conducted in the control (saline-paired) context. In the control context, rats exhibited goal-directed action control; i.e., they were able selectively to withhold the action that previously earned the now devalued outcome. However, these same rats were impaired when tested in the alcohol-paired context, performing both actions at the same rate regardless of the current value of their respective outcomes. Subsequent testing revealed that the rats were capable of overcoming this impairment if they were giving response-contingent feedback about the current value of the food outcomes. These results provide a clear demonstration of the disruptive influence that alcohol-paired cues can exert on decision-making in general and goal-directed action selection and choice in particular.

Keywords: addiction, decision-making, habits, choice

\section{INTRODUCTION}

Addiction may be viewed as a disorder of decision-making. Drug addicts compulsively seek out and use drugs even though such behavior can have devastating effects on their health and livelihood. There is growing recognition among researchers that environmental cues that signal the availability of drugs play a role in supporting pathological drug seeking in active drug users and in triggering relapse in abstaining addicts (Stewart et al., 1984; Tiffany, 1990; O’Brien et al., 1998). However, much remains unknown about how drug-paired cues influence decision-making and behavioral control processes more generally.

One explanation advanced by researchers from diverse theoretical backgrounds is that stimuli associated with drug use acquire the capacity to interfere with cognitive processing (Tiffany, 1990), rational decision-making (Bernheim and Rangel, 2004), or goaldirected action selection (Ostlund and Balleine, 2008). Despite important differences between these individual accounts, this general hypothesis attempts to explain why under certain conditions addicts can sometimes act in their own best interest by abstaining from drug use but have difficulty doing so when confronted with drug-paired cues. Importantly, this view predicts that such cues should interfere with all actions requiring planning or deliberation, not just those relating to drug use. In support of this claim, it has been shown that imagery that provokes cigarette craving in smokers (Sayette and Hufford, 1994; Cepeda-Benito and Tiffany, 1996) or alcohol craving in drinkers (Sayette et al., 1994) can disrupt performance of an unrelated reaction time task that requires cognitive processing.

The current study approached this hypothesis from an animal learning perspective. As with humans, rats can select actions in a voluntary, goal-directed manner. Their tendency to perform an action, like pressing a lever, depends on the current incentive value of the reward that would normally be produced by that action (cf. Balleine and Ostlund, 2007; Balleine et al., 2009 for recent reviews). We assessed whether the presence of contextual cues that signaled alcohol intoxication would disrupt rats' capacity for goal-directed action selection. Rats were first given differential context conditioning such that one context was paired with ethanol injections and a second, distinctive context was paired with saline injections. All rats were then trained in a third, neutral context to press one lever for grain pellets and different lever for sucrose pellets. They were then administered a pair of choice tests, conducted in extinction, to evaluate the rats' ability to adjust their performance of the two actions in response to a reduction in the value of one of the two food outcomes. One test was conducted in the alcohol-paired context and the other was conducted in the control (saline-paired) context. If drug-paired cues interfere with goal-directed decision-making, then rats should be capable of selecting actions based on the value of their anticipated outcomes when tested in the control context but should be impaired in doing so when tested in the ethanol-paired 
context. To further characterize the predicted impairment, we also assessed the effects of ethanol-paired cues on devaluation performance when rats were given response-contingent feedback about the current outcome values.

\section{MATERIALS AND METHODS}

All procedures were approved by UCLA's Animal Research Committee.

\section{SUBJECTS AND APPARATUS}

Adult (90-120 days) Long-Evans rats (Harlan Laboratories) were used as subjects. Rats were housed in pairs in transparent plastic tubs in a temperature- and humidity-controlled vivarium. Experiments were conducted during the light phase of the 12:12-h light/dark cycle. Tap water was always available when rats were in their home cages. During instrumental conditioning and behavioral testing, rats were food-deprived by restricting their daily allotment of food to between 10-14 g of chow per day to maintain them at approximately $85 \%$ of their free-feeding bodyweight. Behavioral procedures were conducted in eight identical Med-Associates operant chambers (standard modular chamber; interior length $30.5 \mathrm{~cm}$, width $24.1 \mathrm{~cm}$, height $21.0 \mathrm{~cm}$ ), situated in sound- and light-attenuated shells. The front and rear walls were composed of aluminum paneling, and the ceiling, sidewall and hinged front door were composed of transparent plexiglass. The floor consisted of 16 stainless steel rods. A waste pan containing corncob bedding was placed under the grid floor. Each chamber had a pair of retractable levers positioned to the left and right of a recessed food magazine, into which grain and sucrose food pellets (Bioserv; $45 \mathrm{mg}$ ) could be dispensed. An infrared beam crossed the magazine opening, allowing for the detection of head entry responses. Each chamber was also equipped with three infrared beams positioned $5 \mathrm{~cm}$ above the grid floor across the length of chamber (one centered and others $5 \mathrm{~cm}$ from each side wall) to measure activity in the horizontal plane. A houselight located on the wall opposite the levers was used to illuminate the chamber throughout all behavioral training/ testing sessions.

The above description refers to the bare chamber, which served at the training context during instrumental conditioning sessions. During context conditioning and outcome devaluation test sessions, we added visual, tactile, and olfactory cues to create two distinctive contexts. For Context A, panels with black-and-white horizontal stripes were positioned outside the transparent sidewall and door, a plexiglass sheet covered with black, sandpaper-textured adhesive-backed material (grip tape) covered the grid floor, and a paper towel scented with $0.5 \mathrm{ml}$ of a $10 \%$ artificial vanilla extract solution (McCormick and Co. Inc., Baltimore, MD, USA) was placed in the waste pan (below the floor). For Context B, white panels with black filled circles were placed outside the sidewall and door, the floor was covered with a white, diamond-textured acrylic sheet, and a paper towel scented with $0.5 \mathrm{ml}$ of $10 \%$ acetic acid was placed in the waste pan.

\section{CONTEXT CONDITIONING}

For rats in Experiment $1(N=12)$, one context (either A or B) was paired with an injection of ethanol solution $(1 \mathrm{~g} / \mathrm{kg} ; 20 \%$ ethanol in saline; i.p.) and the other context was paired with an injection of an equivalent volume of buffered saline solution. Rats were exposed to the two contexts in alternation over a period of 14 days (seven exposures to each context). During context exposure sessions, rats were placed in the context for $10 \mathrm{~min}$, at which point they were removed from the chamber and administered an injection of the appropriate solution (either ethanol or saline). They were then returned to the context for $60 \mathrm{~min}$ before being returned to their home cage. A second group of rats (Experiment 2) was given identical treatment except they received saline in both contexts. This saline-only control group $(N=12)$ was used to assess the characteristics of instrumental performance in rats that had no experience with alcohol.

\section{INSTRUMENTAL CONDITIONING}

The food deprivation regimen was initiated $24 \mathrm{~h}$ after the last session of context conditioning and was maintained throughout the rest of the experiment. Rats were allowed to rest in their home cage for 5 days between context conditioning and instrumental conditioning. Two sessions of magazine training were then conducted. During each session, 20 grain pellets and 20 sucrose pellets were delivered according to a random time-30-s schedule. The rats were then given 7 days of instrumental training. For the first 6 days of training the two lever press actions were rewarded in separate daily sessions with different outcomes. For half of the subjects in each group, pressing the left lever earned grain pellets and pressing the right lever earned sucrose pellets, whereas the remaining subjects were trained using the opposite response-outcome relationships. Each session started with the illumination of the house light and the insertion of the left or right lever (session order was alternated over days) and ended after 45 min or 30 pellets had been earned, whichever came first. On the first 2 days of training, lever pressing was continuously reinforced, such that every lever press produced the appropriate outcome. The schedule of reinforcement was then shifted to random ratio (RR)- 5 for 2 days before being shifted to a RR-10 schedule for the rest of training. During the last day of instrumental conditioning rats were trained with the same two action-outcome contingencies in alternating blocks within a single session. At the beginning of the session, one of the two levers (randomly determined for each rat) was inserted into the chamber. Lever pressing was reinforced on a RR-10 schedule until five outcomes were earned, at which point the lever was retracted from the chamber. Three minutes later the other lever was inserted until the rat had earned five presentations of the other outcome. This cycle was repeated twice more, until each outcome had been earned 15 times. This alternation training procedure was also used to reestablish responding between outcome devaluation test sessions.

\section{DEVALUATION TESTING}

Rats were given two outcome devaluation tests to assess their ability to respond in a goal-directed manner in the presence and in the absence of alcohol-paired contextual cues. Outcome devaluation was induced using a specific satiety procedure. Rats were given $60 \mathrm{~min}$ of unrestricted access to one of the two training outcomes in their home cage immediately before each of the two tests. Half of the rats in each group were sated on grain pellets and half were sated on sucrose pellets, counterbalanced across context conditioning and instrumental conditioning treatments. They were then placed in the behavioral chamber, which was prepared as Context A or B. Each 
test began with a 10 -min context exposure period with the levers retracted. The levers were then inserted for $5 \mathrm{~min}$. Responses were recorded but were not reinforced. After a session of retraining on the two levers, rats were administered a second devaluation test that was identical to the first except they were tested in the opposite context. For half of the rats in each group, Test 1 was conducted in Context $A$ and Test 2 was conducted in Context B, whereas the other rats were tested in the opposite order. Test order was counterbalanced across earlier treatments. After another session of retraining with the two action-outcome relationships, the rats were administered a final test to assess whether alcohol-paired cues affected the expression of goal-directed action selection when response-contingent feedback about goal value was provided. The rats were given $60 \mathrm{~min}$ of access to the same outcome that was devalued in previous tests before being placed in the chamber. For all alcohol-treated subjects (Experiment 1), the chamber was prepared as the context that was paired with alcohol. For saline-treated controls (Experiment 2), the chamber was prepared as Context A or B, counterbalanced across earlier treatments. After $10 \mathrm{~min}$ of context exposure, the two levers were inserted into the chamber for $30 \mathrm{~min}$ and rats were allowed to earn the two outcomes by lever pressing. Outcomes were delivered contingently according to the action-outcome relationships used during training, however a modified progressive ratio schedule of reinforcement was used to encourage responding and sampling of both action-outcome contingencies. For each action, the appropriate outcome was delivered after the first, third, sixth, 10th, and 15 th lever press. All subsequent responses were then reinforced according to a RR-10 schedule.

\section{BEHAVIORAL MEASURES}

Locomotor activity during context conditioning sessions and outcome devaluation tests was quantified as the total number of breaks in three horizontal infrared beams. Instrumental performance during outcome devaluation testing was expressed as a percentage of baseline responding by dividing the response rate (presses per minute) at test by the average response rate on that lever during the most recent session of instrumental training.

\section{RESULTS}

\section{CONTEXT CONDITIONING}

The results from the context conditioning phase of Experiment 1 are presented in Figure 1. Panel A shows locomotor activity during the 10-min period before each injection. Rats decreased their activity in both the ethanol- and saline-paired contexts over days, presumably due to habituation of the exploratory response to these cues. A two-way repeated-measures ANOVA with drug (ethanol vs. saline) and session (1-7) as factors found a significant effect of session $\left(F_{6,66}=10.60 ; p<0.001\right)$, but found no effect of drug $\left(F_{1,11}=0.38 ; p=0.55\right)$ or a drug by session interaction $\left(F_{6,66}=1.77\right.$; $p=0.12$ ). As can be seen in Panel $B$, which plots activity during the 60-min post-injection period, rats displayed significantly lower levels of locomotor activity after receiving ethanol injections than after saline injections. This effect was relatively stable over sessions. A two-way repeated-measures ANOVA with drug (ethanol vs. saline) and session (1-7) as factors detected a significant main effect of drug $\left(F_{1,11}=19.29 ; p<0.001\right)$, a significant effect of sessions $\left(F_{6,66}=2.22 ; p=0.05\right)$, but found no interaction between these factors $\left(F_{6,66}=0.90 ; p=0.50\right)$.

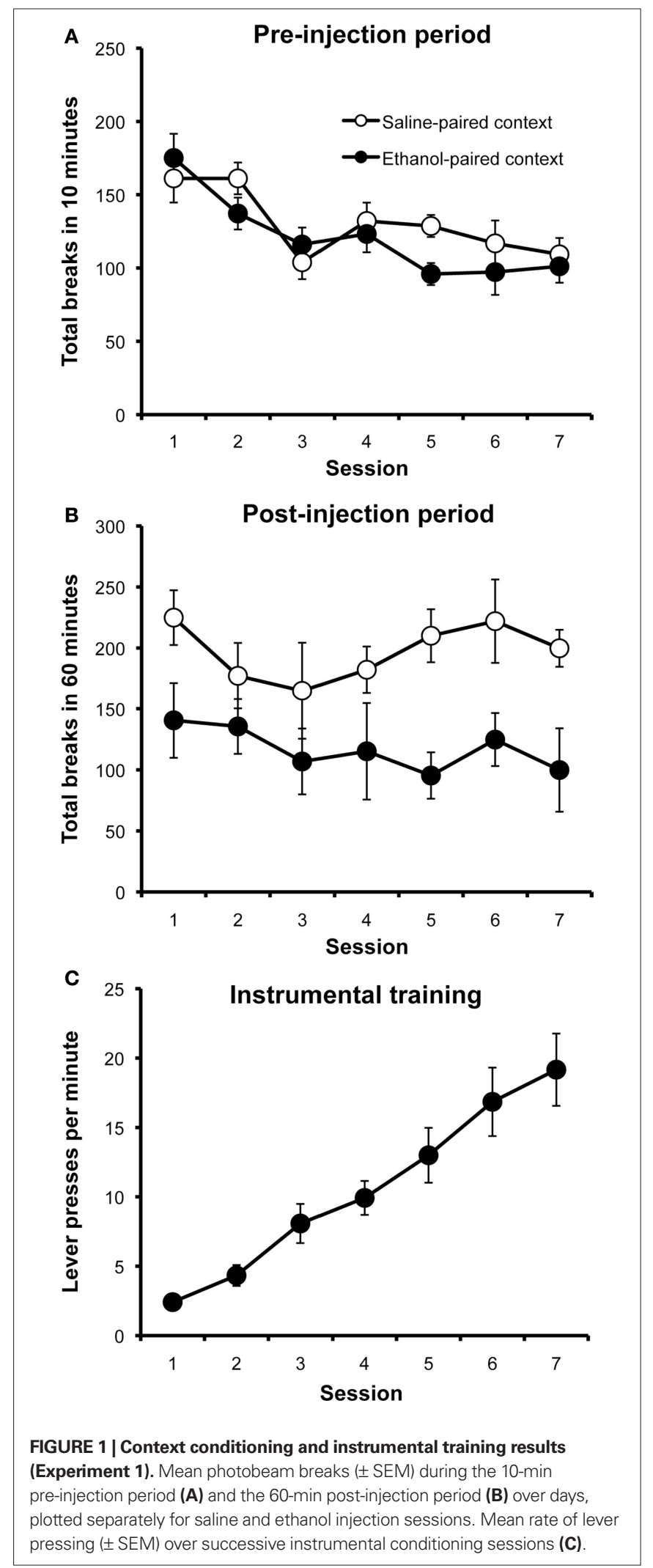

INSTRUMENTAL TRAINING

Figure 1C presents the data from instrumental training sessions for Experiment 1, plotted as the mean number of lever presses performed per minute over days, averaged across the two actions. 
All rats in this study had received the ethanol-treatment and all successfully learned to lever press, increasing their rate of responding over sessions as the response requirement was increased (main effect of session: $F_{1,11}=37.58 ; p<0.001$ ). The response rate for the action whose outcome would be devalued at test (Mean 20.62; SEM 3.08) did not significantly differ from that for the action whose outcome would not be devalued (Mean 18.19; SEM 2.36) $\left(F_{1,11}=1.31 ; p=0.28\right)$.

\section{DEVALUATION TESTING: NO FEEDBACK}

To assess whether ethanol-paired cues disrupt goal-directed action selection, rats in Experiment 1 were administered two outcome devaluation tests, one conducted in the ethanol-paired context and the other in the saline-paired context. These tests were given in extinction (i.e., without explicit feedback about outcome value) and, as such, the rats' choice between actions depended on their memory of the action-outcome relationships and of the updated values for those outcomes. The results are presented in Figure 2, plotted separately for the test conducted in ethanol-paired context (Panel A) and the test conducted in the saline-paired context (Panel B). Rats exhibited goal-directed choice in the saline-paired context, suppressing their performance of the action that had earned the devalued outcome while continuing to perform the other, non-devalued action at a high rate. However, consistent with the hypothesis that drug-related cues interfere with the expression of goal-directed action selection, the same rats failed to show sensitivity to outcome devaluation when tested in the ethanol-paired context. A three-way ANOVA using context (ethanol-paired vs. saline-paired), action (devalued

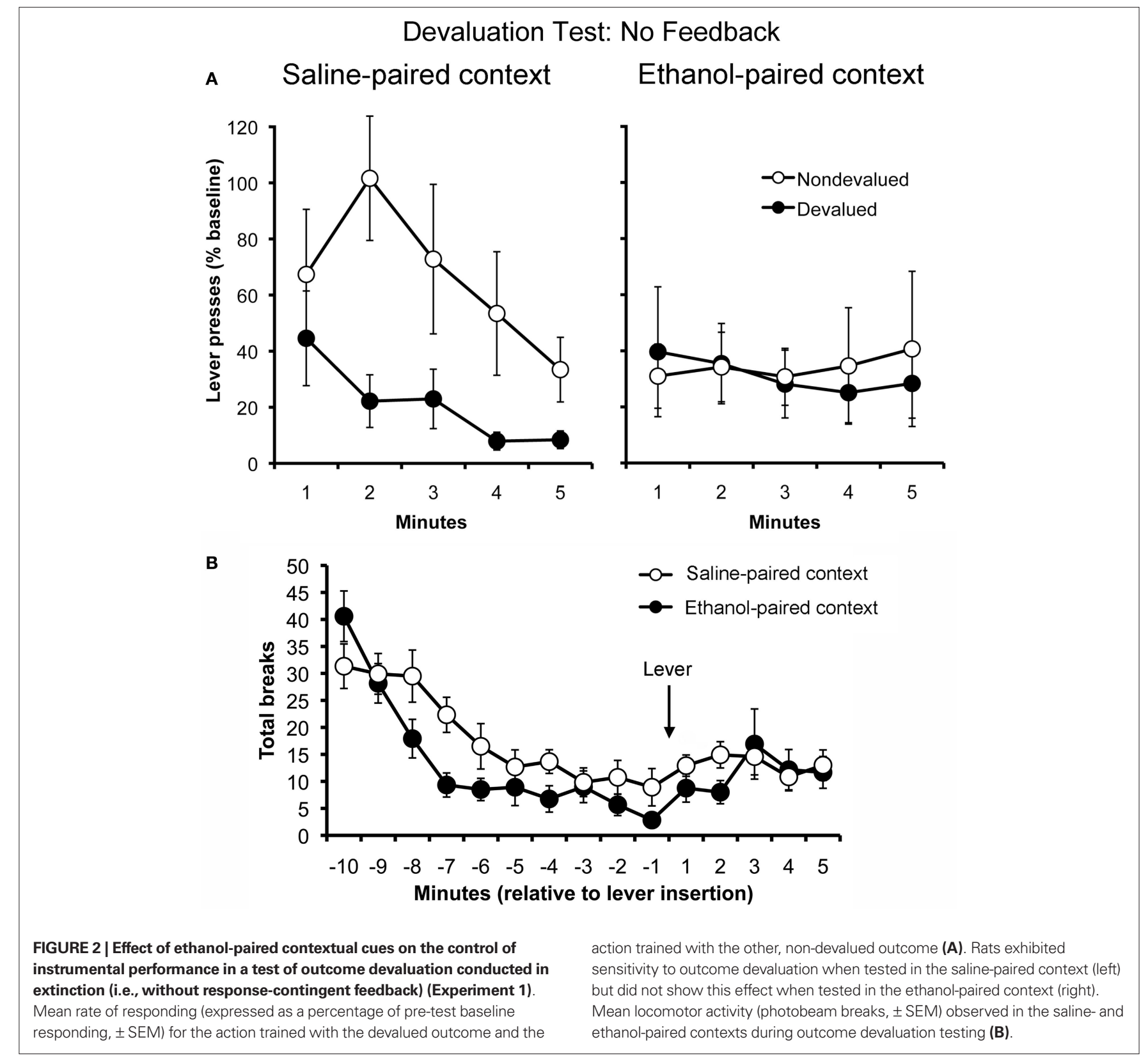


vs. non-devalued) and time ( $\min 1-5)$ as factors found a significant main effect of time $\left(F_{4,44}=4.52 ; p=0.004\right)$. The main effects of action $\left(F_{1,11}=4.04 ; p=0.07\right)$ and context $\left(F_{1,11}=2.01 ; p=0.18\right)$ were not significant. The action by time $\left(F_{4,44}=0.71 ; p=0.59\right)$, context by time $\left(F_{4,44}=2.33 ; p=0.07\right)$, and context by action by time $\left(F_{4,44}=0.45 ; p=0.77\right)$ interactions also failed to reach significance. Importantly, however, there was a significant interaction between context and action $\left(F_{1,11}=4.76 ; p=0.05\right)$, confirming that the rats' choice between actions depended on which context they were in at test. To further explore this effect, separate action by time ANOVAs were conducted for each of the two context conditions. When tested in the saline-paired context, the rats displayed sensitivity to outcome devaluation (main effect of action: $F_{1,11}=5.31 ; p=0.04$ ) and showed a significant reduction in responding over time $\left(F_{4,44}=5.64 ; p=0.001\right)$ due to extinction. However, when the tested in the ethanol-paired context, the same rats showed no preference between the two actions $\left(F_{1,11}=0.10 ; p=0.76\right)$ and failed to significantly decrease their rate of responding over minutes $\left(F_{4,44}=0.19 ; p=0.94\right)$, which suggests that these cues were also effective in disrupting the sensitivity of instrumental performance to extinction. The action by time interaction did not reach significance in either context (saline-paired context: $F_{4,44}=1.25 ; p=0.30$, ethanol-paired context: $F_{4,44}=0.10 ; p=0.98$ ). Taken together, these findings demonstrate that environmental cues that signal alcohol intoxication can acquire the ability to transiently disrupt goal-directed action selection.

Figure 2C presents the rats' locomotor activity during these tests. Activity measures were taken starting 10-min before the levers were inserted and finished after the 5-min test period. Levels of activity were suppressed in the ethanol-paired context, relative to activity in the saline-paired context. This effect was particularly clear during the initial $10 \mathrm{~min}$ of the test, before the rats were allowed to perform the lever press response. A repeated-measures ANOVA performed on these data (first 10 bins) found a significant effect of time $\left(F_{9,99}=22.06 ; p<0.001\right)$, and a significant effect of context $\left(F_{1,11}=4.64 ; p=0.05\right)$. More importantly, we found a significant time by context interaction $\left(F_{9,99}=2.70 ; p=0.007\right)$, indicating that the rats' pattern of activity over time differed across the two contexts. During the last $5 \mathrm{~min}$, which covered the test of lever press performance, the suppressive effects of the ethanol-paired context were much less apparent (see Figure 3C). Statistical analysis of these data (last five bins) found no effect of time $\left(F_{1,11}=0.34 ; p=0.57\right)$ or context $\left(F_{4,44}=1.26 ; p=0.30\right)$, and found no interaction between these factors $\left(F_{4,44}=0.77 ; p=0.55\right)$.

\section{OUTCOME DEVALUATION TESTING: WITH FEEDBACK}

The results of the first pair of outcome devaluation tests demonstrate that ethanol-paired environmental cues can disrupt goal-directed action selection. Importantly, no outcomes were delivered during these tests, requiring subjects to rely on their memory of the actionoutcome contingencies that were present during training in order to adjust their behavior in response to the change in outcome value. Thus, the insensitivity to outcome devaluation found in the ethanolpaired context may have been the result of a failure to retrieve the appropriate action-outcome associations and/or a reliance on an alternative response selection strategy (e.g., one based on stimulusresponse, or habit, learning). In either case, one might expect rats to overcome this impairment if given response-contingent outcome

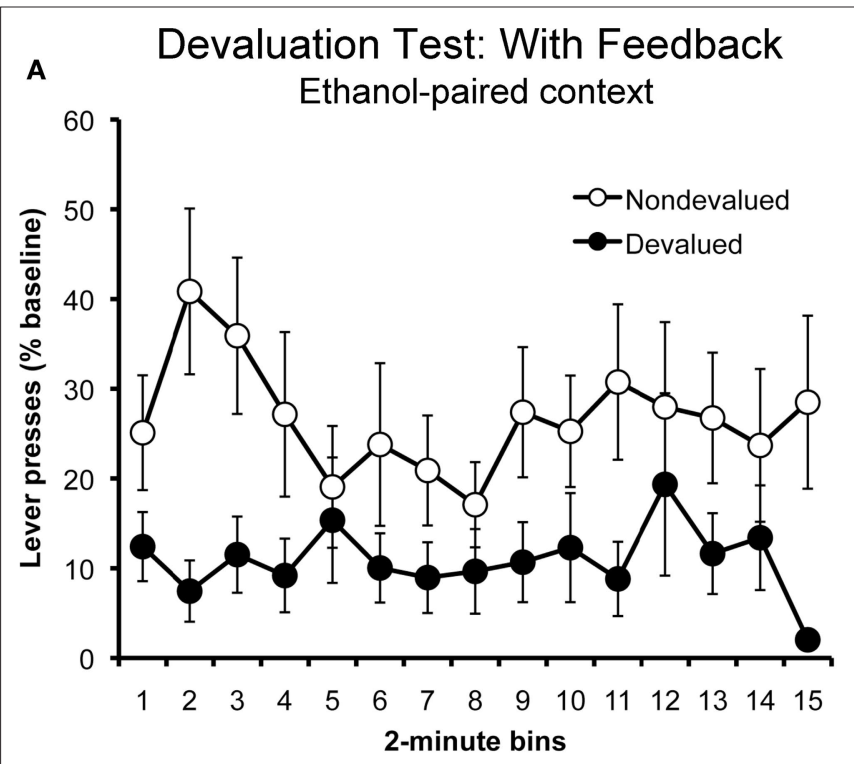

B

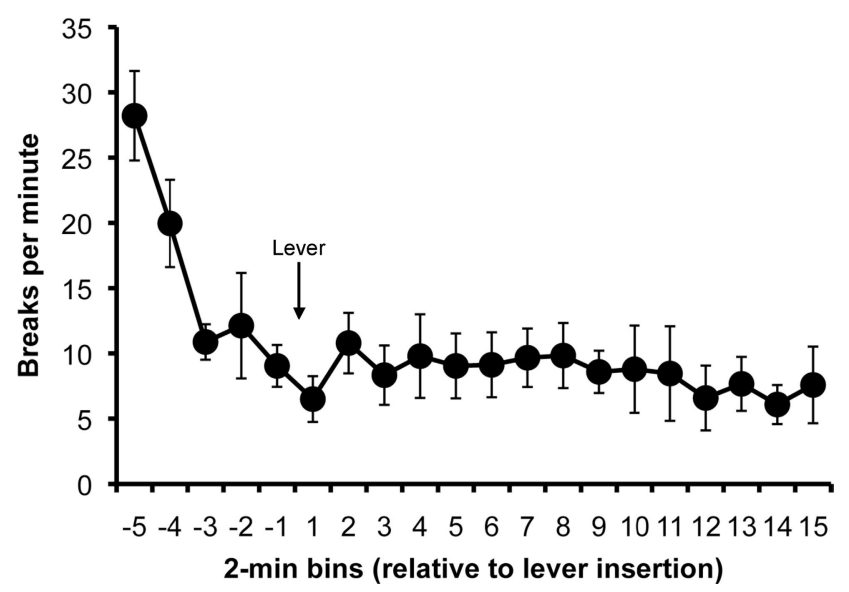

FIGURE 3 | Sensitivity to outcome devaluation in the ethanol-paired context in the rewarded test (i.e., with response-contingent feedback) (Experiment 1). Mean rate of lever pressing (percentage of baseline, \pm SEM) for the devalued and non-devalued outcomes (A). Rats displayed a selective suppression in their performance of the action that earned the devalued outcome at test, despite the presence of ethanol-paired cues. Mean locomotor activity (photobeam breaks, \pm SEM) observed during the rewarded outcome devaluation test (B)

deliveries, providing them with immediate feedback about the relative value of the two rewards. Alternatively, it is possible that cues signaling alcohol promote compulsive behavior, preventing subjects from considering the consequences of their actions, even when those consequences are experienced in a response-contingent manner (see Section "Discussion"; Ostlund and Balleine, 2008). To assess these two possibilities, all rats in Experiment 1 were given a final outcome devaluation test in the ethanol-paired context, this time with each action earning the appropriate outcome, as during initial training.

The data from this test are presented in Figure 3. Panel A plots the rate of responding for the actions that produced the devalued and non-devalued outcomes. Unlike when they were tested 
without feedback, rats tested in the presence of ethanol-paired cues exhibited sensitivity to outcome devaluation when the training outcomes were contingently delivered. A two-way repeated-measures ANOVA with action and time as factors found a significant effect of action $\left(F_{1,11}=12.65 ; p=0.005\right)$, but found no effect of time $\left(F_{14,154}=1.03 ; p=0.42\right)$ and no action by time interaction $\left(F_{14,154}=0.68 ; p=0.79\right)$.

Panel B plots the rats' locomotor activity at test during the $10 \mathrm{~min}$ before and $30 \mathrm{~min}$ after the levers were inserted into the chamber, plotted in 2-min bins. Activity levels appeared to decrease rapidly before the lever was inserted (first five bins). Analysis of these data using a repeated-measures ANOVA found a significant main effect of time $\left(F_{4,44}=11.07 ; p<0.001\right)$. Activity levels appeared to remain relatively stable while the rats lever pressed. An ANOVA conducted on these data (last 15 bins) found no effect of time $\left(F_{14,154}=0.69 ; p=0.78\right)$.

\section{SALINE-ONLY CONTROL EXPERIMENT}

The results presented above support the hypothesis that environmental cues that have been paired with alcohol intoxication disrupt the processes that mediate voluntary, goal-directed action selection. However, this interpretation assumes that normal, ethanol-naïve rats would rely on a goal-directed action selection strategy under the current training and testing conditions. To characterize the instrumental performance of ethanol-naïve subjects, a second group of rats underwent the same context conditioning procedures as ethanol-treated rats but received saline injections in both contexts. They were then trained on the two action-outcome contingencies, as in the previous experiment. One rat failed to complete instrumental training and was excluded from the experiment $(N=11)$. By the last day of training, the remaining rats reached response rates comparable to ethanoltreated rats, and their rates of responding for the to-be devalued (Mean 14.95; SEM 2.46) and non-devalued outcomes (Mean 18.82; SEM 4.31) did not significantly differ $\left(F_{1,10}=2.26 ; p=0.16\right)$.

Figure 4 presents the results of outcome devaluation testing. The results from the tests conducted without response-contingent feedback are displayed in Panel A. Because both contexts were paired with saline for all subjects, the data have been collapsed across tests. As expected, the saline-only control group displayed clear evidence of goal-directed action selection, selectively withholding their performance of the action that, in training, had produced the outcome that was devalued at test, relative to their performance of the other action. A two-way repeated-measures ANOVA using action and time as factors found a significant effect of action $\left(F_{1,10}=8.77\right.$; $p=0.01)$ and of time $\left(F_{4,40}=3.94 ; p=0.009\right)$. The action by time interaction $\left(F_{4,40}=2.46 ; p=0.06\right)$ failed to reach significance. This finding is important because it establishes that alcohol-naïve rats were capable of learning the relevant action-outcome associations during training and were able to generalize these relationships across contexts to guide their action selection at test.

The results of the outcome devaluation test conducted with response-contingent feedback are presented in Panel B. Not surprisingly, the saline-only treated rats continued to display sensitivity to outcome devaluation when their actions actually produced the devalued and non-devalued outcomes. A two-way repeatedmeasures ANOVA using action and time as factors detected a significant effect of action $\left(F_{1,10}=6.67 ; p=0.03\right)$ and of time $\left(F_{14,140}=4.71\right.$;

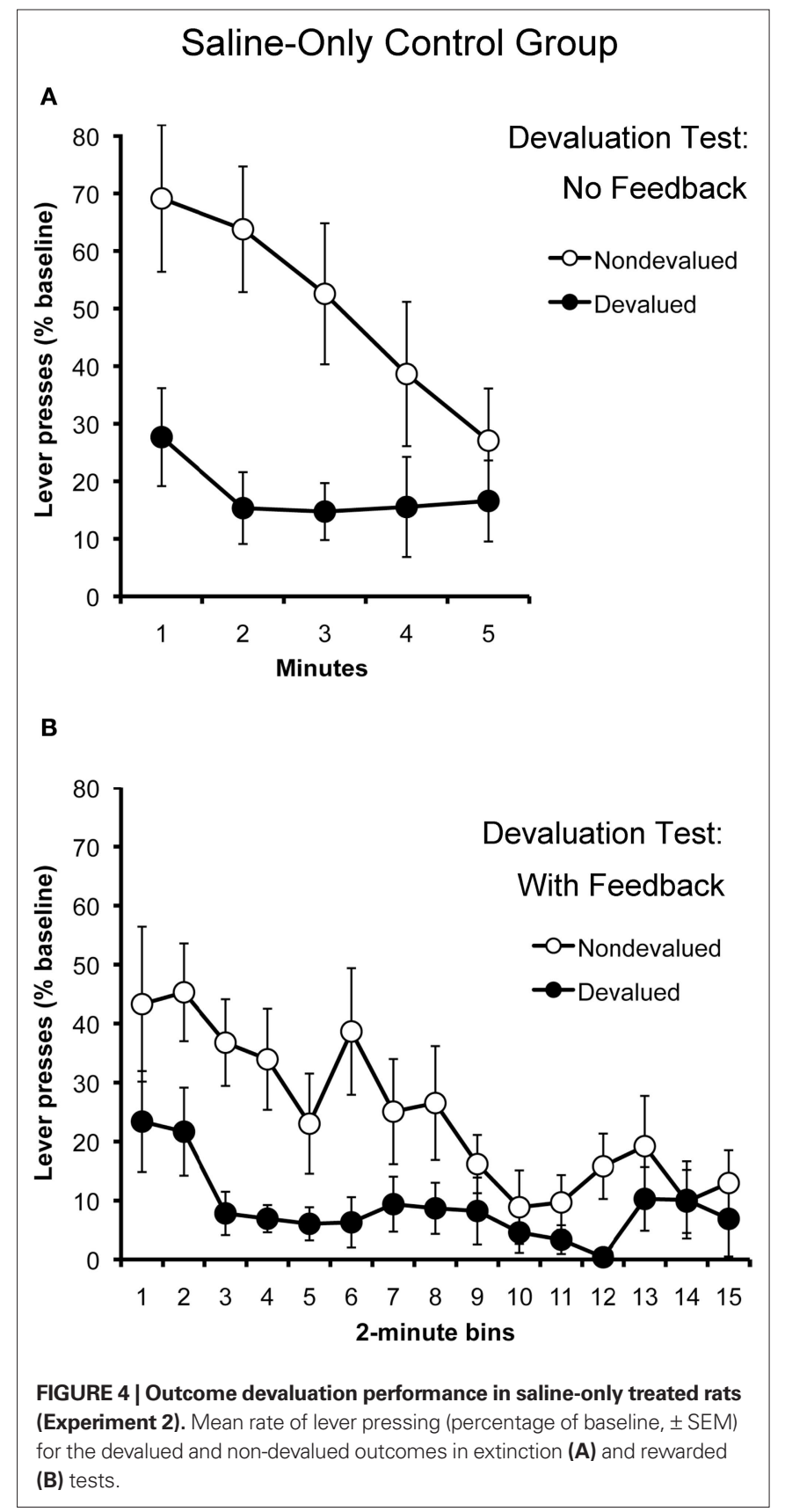

$p<0.001)$. The action by time interaction $\left(F_{14,140}=1.582 ; p=0.09\right)$ was not significant. The main effect of time suggests that the rats in this experiment became sated on both rewards over the course of the 30-min test, further reducing their rate of responding on both actions. This is noteworthy because the rats in Experiment 1 showed no effect of time when given a rewarded test in the alcohol-paired context. Although one should be cautious when comparing results across experiments, this finding suggests that the disruptive effects of alcohol-paired cues on action selection may not be entirely reversed by providing subjects with response-contingent feedback.

More importantly, however, the findings of Experiment 2 suggest that alcohol-naïve rats tend to use a goal-directed strategy when selecting instrumental actions under the current training and 
testing conditions. Therefore it is reasonable to conclude that the impaired outcome devaluation displayed by ethanol-treated rats in the ethanol-paired context reflects a disruption of an action selection strategy that normally would have mediated their behavior.

\section{DISCUSSION}

The current study demonstrates that alcohol-paired environmental cues can disrupt decision-making; rats tested in a context that signaled alcohol intoxication were found to lack the capacity to select actions based on their anticipated outcomes. Importantly, these rats were able to select their actions in a goal-directed manner when tested in a different context that had not been paired with alcohol, indicating that the influence of the alcohol-paired context on action selection was transitory, and was therefore unlikely to be the product of persistent neuroadaptions induced by the alcohol exposure regimen. The finding that the alcohol-paired context failed to disrupt goal-directed performance when rats were given response-contingent feedback about the current value of the available outcomes provided further support for this conclusion. This pattern of findings is therefore consistent with the hypothesis, presented in the introduction, that cues associated with drugs or alcohol can exert an acute, time-limited influence over decisionmaking, interfering with the cognitive processes that support goaldirected action selection and choice.

Although we found that ethanol-paired cues rendered instrumental performance insensitive to outcome devaluation, it is not clear how these cues have their effect. It has been argued that drugrelated cues tax limited cognitive resources which are essential for non-automatic decision-making, allowing automatic processes to control performance (Tiffany, 1990). A similar distinction is made in the field of instrumental conditioning, where it has been shown that rats tend to select actions using either a goal-directed strategy based on action-outcome learning or a habit strategy based on stimulus-response learning (see Yin et al., 2008). Working from this perspective, we have argued (Ostlund and Balleine, 2008) that drugpaired cues might cause a transition from goal-directed to habitual control, leaving instrumental performance insensitive to outcome devaluation, an account that fits nicely with the results reported here. Alternatively, rather than encouraging habitual performance, it is possible that the alcohol-paired cues interfered with retrieval or implementation of action-outcome associations, preventing rats from using the current value of the two rewards to guide their choice of actions. A third possibility is that the presence of cues signaling alcohol intoxication caused a reappraisal of the two food outcomes such that both outcomes were assigned equivalent, low incentive values (e.g., by producing anhedonia). However, this account is not consistent with our finding that rats exhibited sensitivity to outcome devaluation when they were given response-contingent feedback; this treatment should only have strengthened their assessment that both outcomes were currently undesirable.

It is important to note that rats given pairings between environmental cues and peripheral ethanol injections have been shown to develop a conditioned aversion to those cues (Cunningham, 1979, 1981; van der Kooy et al., 1983; Bormann and Cunningham, 1998). Our experimental preparation prevented us from directly measuring our rats' preference between the ethanol- and saline-paired contexts, but we did find that they displayed significantly lower levels of locomotor activity in the ethanol-paired context at test, which recent findings suggest may be indicative of conditioned place aversion learning (Hill et al., 2007). It is therefore prudent to consider the possibility that an ethanol-induced conditioned place aversion may have generated a competing behavior (e.g., freezing) that interfered with the expression of instrumental performance, making it difficult for us to evaluate whether this behavior was sensitive to outcome devaluation or not. However, there are several features of our results that are incompatible with this possibility. First, although the ethanolpaired context was effective in suppressing activity at test, this effect was limited to the period before the lever insertion; no differences in activity were observed when the rats were actually pressing the levers. Second, although the mean rate of lever pressing was numerically lower in ethanol-paired context than in the saline-paired context, this effect was not significant. Third, since response rates were not at the behavioral floor (i.e., no presses on either lever) in the ethanolpaired context, any competing response that did exist should not have prevented the rats from displaying an outcome devaluation effect, which involves selectively withholding whichever action had earned the devalued outcome. Thus, even if the rats had responded at a significantly lower rate in the ethanol-paired context, there was sufficient responding to observe whether their choice between the two levers was guided by goal-directed action selection.

Although this conditioned aversion-induced response competition account does not adequately explain the current results, it is possible that aversive conditioning played some role in mediating the influence of alcohol-paired cues over instrumental performance. Recent studies in rodents (Dias-Ferreira et al., 2009) and humans (Schwabe and Wolf, 2009, in press) have demonstrated that stress can promote habitual performance and/or interfere with goal-directed action selection. Therefore, it is possible that the disruption of goal-directed action selection produced by the context paired with alcohol in the current study was mediated by its tendency to evoke an acute stress response. This interpretation is consistent with the widely held view that stress contributes to drug relapse (Sinha, 2001; Lê and Shaham, 2002; Goeders, 2003) and offers a novel behavioral mechanism through which it could be exerting its effects. However, as the current study was not designed to evaluate this hypothesis, further research will be needed to determine what, if any, role stress plays in mediating the disruptive effects of alcohol-paired cues on instrumental action selection.

Recent years have seen considerable interest in theories of addiction that attempt to explain the compulsive nature of drug seeking through the effects of chronic drug use on brain systems responsible for normal learning and memory. Some have suggested that drug use leads to exaggerated habit formation, promoting the use of stimulus-guided rather than outcome-guided action selection in addicts (Tiffany, 1990; Berke and Hyman, 2000; Everitt et al., 2001). Indeed, rats given repeated psychostimulant administration prior to instrumental conditioning with natural food rewards have been shown to display accelerated habit formation, indicating that such treatment results in long-lasting adaptation in the neural circuitry underlying this form of learning (Nelson and Killcross, 2006). In contrast, the incentive sensitization theory of addiction (Robinson and Berridge, 1993) proposes that repeated drug exposure results in permanent alterations in the neural circuitry responsible for incentive motivation, which, under normal conditions, allows stimuli associ- 
ated with natural rewards and drugs to elicit approach behavior and invigorate reward seeking. Drug-induced adaptations in this system result in a sensitization of incentive motivational processes, potentiating the excitatory influence that reward- and drug-paired cues have over behavior (Wyvell and Berridge, 2001). Although both accounts have received strong experimental support over the years, neither can explain the acute and time-limited disruption of goal-directed action selection produced by ethanol-paired stimuli reported here. The accelerated habit learning account predicts that if our repeated alcohol pre-exposure regimen were to have any effect at all on instrumental performance, it should have resulted in a persistent, contextindependent disruption of goal-directed performance. In contrast, according to the incentive sensitization account, one might expect the presence of ethanol-paired cues to invigorate responding by activating the incentive motivational system, which has presumably been sensitized due to the repeated alcohol administration. Not only did we fail to find such an effect, this account does not directly address the insensitivity of action selection to outcome devaluation. Even if both actions should have been performed at a higher rate (relative to baseline conditions or when rats were tested in the saline-paired context), one would still expect to observe higher rates of responding on the action whose outcome remained valuable.

The current results may be particularly relevant to the early stages of the addiction process. Experienced drug users are said to display compulsive drug seeking if this behavior persists despite its

\section{REFERENCES}

Balleine, B. W., and Ostlund, S. B. (2007). Still at the choice-point. Ann. N.Y. Acad. Sci. 1104, 147-171.

Balleine, B. W., Liljeholm, M., and Ostlund, S.B. (2009). The integrative function of the basal ganglia in instrumental conditioning. Behav. Brain Res. 199, 43-52.

Berke, J. D., and Hyman, S. E. (2000). Addiction, dopamine, and the molecular mechanisms of memory. Neuron 25, 515-532.

Bernheim, B. D., and Rangel, A. (2004). Addiction and cue-triggered decision processes. Am. Econ. Rev. 94, 1558-1590.

Bormann, N. M., and Cunningham, C. L. (1998). Ethanol-induced conditioned place aversion in rats: effect of interstimulus interval. Pharmacol. Biochem. Behav. 59, 427-432.

Cepeda-Benito, A., and Tiffany, S. (1996). The use of a dual-task procedure for the assessment of cognitive effort associated with cigarette craving. Psychopharmacology 127, 155-163.

Cunningham, C. L. (1979). Flavor and location aversions produced by ethanol1. Behav. Neural Biol. 27, 362-367.

Cunningham, C.L. (1981). Spatial aversion conditioning with ethanol. Pharmacol. Biochem. Behav. 14, 263-264.

Dias-Ferreira, E., Sousa, J. C., Melo, I., Morgado, P., Mesquita,A.R., Cerqueira, J. J., Costa, R. M., and Sousa, N. (2009). Chronic stress causes frontostriatal

considerable adverse consequences. As argued elsewhere (Ostlund and Balleine, 2008), this insensitivity to response-contingent punishment suggests that compulsive drug seeking may be dominated by habitual control; such addicts lack the capacity to shift from habitual to goal-directed performance when confronted by aversive stimuli. However, the current findings demonstrate that drug-paired cues can exert a more subtle, time-limited effect on instrumental control. Although ethanol-paired cues were found to disrupt the rats' tendency to selectively withhold an action whose outcome had been devalued if tested without feedback (i.e., in extinction), this effect was not observed when the devalued outcome was delivered at test, which may be considered a form of punishment. Thus it appears that context-alcohol learning can interfere with goal-directed action selection without generating truly compulsive behavior. However, even if transient and rapidly overcome, it is possible that exposure to drug-related cues could promote drug use by obstructing the user from considering the consequences of this behavior, thereby rendering action initiation more impulsive. This increased drug exposure could, as a consequence, result in more persistent neuroadaptions, facilitating the transition from drug abuse to addiction.

\section{ACKNOWLEDGMENTS}

This research was supported by grant AA18014 from NIAAA to Bernard Balleine and training fellowship T32 MH17140 to Sean Ostlund.

on cognitive resources in smokers. $J$. Abnorm. Psychol. 103, 812-818.

Sayette, M.A., Monti, P. M., Rohsenow, D. J., Gulliver, S. B., Colby, S. M., Sirota, A. D., Niaura, R., and Abrams, D. B. (1994). The effects of cue exposure on reaction time in male alcoholics. J. Stud. Alcohol 55, 629-633.

Schwabe, L., and Wolf, O. T. (2009). Stress prompts habit behavior in humans. J. Neurosci. 29, 7191-7198.

Schwabe, L., and Wolf, O. T. (in press). Socially evaluated cold pressor stress after instrumental learning favors habits over goal-directed action. Psychoneuroendocrinology Available at: http://www.sciencedirect.com/ science/article/B6TBX-4Y52J5T-1/2/ 61b55366b53857949b609e4f6bb173 28 [Accessed April 18, 2010].

Sinha, R. (2001). How does stress increase risk of drug abuse and relapse? Psychopharmacology (Berl.) 158, 343-359.

Stewart, J., De Wit, H., and Eikelboom, R. (1984). Role of unconditioned and conditioned drug effects in the selfadministration of opiates and stimulants. Psychol. Rev. 91, 251-268.

Tiffany, S. T. (1990). A cognitive model of drug urges and drug-use behavior: role of automatic and nonautomatic processes. Psychol. Rev. 97, 147-168.

van der Kooy, D., O’Shaughnessy,M.,Mucha, R.F., and Kalant,H.(1983).Motivational properties of ethanol in naive rats as studied byplaceconditioning.Pharmacol. Biochem. Behav. 19, 441-445.

Wyvell, C. L., and Berridge, K. C. (2001). Incentive sensitization by previous amphetamine exposure: increased cue-triggered "Wanting" for sucrose reward. J. Neurosci. 21, 7831-7840.

Yin, H. H., Ostlund, S. B., and Balleine, B. W. (2008). Reward-guided learning beyond dopamine in the nucleus accumbens: the integrative functions of cortico-basal ganglia networks. Eur. J. Neurosci. 28, 1437-1448.

Conflict of Interest Statement: The authors declare that the research was conducted in the absence of any commercial or financial relationships that could be construed as a potential conflict of interest.

Received:05 May 2010; paperpending published: 17 June 2010; accepted: 25 June 2010; published online: 19 July 2010.

Citation: Ostlund SB, Maidment NT and Balleine BW (2010) Alcohol-paired contextual cues produce an immediate and selective loss of goal-directed action in rats. Front. Integr. Neurosci. 4:19. doi: 10.3389/ fnint.2010.00019

Copyright (c) 2010 Ostlund, Maidment and Balleine. This is an open-access article subject to an exclusive license agreement between the authors and the Frontiers Research Foundation, which permits unrestricted use, distribution, and reproduction in any medium, provided the original authors and source are credited. 